Short Communication

\title{
Drug-induced changes in action potential duration are proportional to action potential duration in rat ventricular myocardium
}

\author{
László Bárándii ${ }^{1}$, Gábor Harmati ${ }^{1}$, Balázs Horváth ${ }^{1}$, Norbert Szentandrássy ${ }^{1}$, János Magyar ${ }^{1}$, \\ András Varró², Péter P. Nánási ${ }^{1}$ and Tamás Bányász ${ }^{1}$ \\ ${ }^{1}$ Department of Physiology, University of Debrecen, Debrecen, Hungary \\ 2 Department of Pharmacology and Pharmacotherapy, University of Szeged, Szeged, Hungary
}

\begin{abstract}
Several cardioactive agents exhibit direct or reverse rate-dependent effects on action potential duration (APD) depending on the experimental conditions. Recently, a new theory has been proposed, suggesting that the reverse rate-dependent mode of drug-action may be a common property of canine, rabbit, guinea pig and human cardiac tissues, and this phenomenon is based on the dependence of drug-action on baseline APD. The aim of the present work was to examine the limitations of this hypothesis by studying the APD lengthening effect of $\mathrm{K}^{+}$channel blockers and the APD shortening effect of $\mathrm{Ca}^{2+}$ channel blockers during the electrical restitution process of rat ventricular action potentials. Rat ventricular muscle was chosen because it has a set of ion currents markedly different from those of other species, its APD is shorter by one order of magnitude than that of the "plateau-forming" larger mammals, and most importantly, its APD increases at higher heart rates - opposite to many other species. The restitution of APD was studied as a function of the diastolic interval, a parameter indicating the proximity of action potentials. It was found that druginduced APD changes in rat myocardium are proportional with the pre-drug value of APD but not with the diastolic interval, indicating that not the proximity of consecutive action potentials, but the baseline APD itself may determine the magnitude of drug-induced APD changes.
\end{abstract}

Key words: Action potential duration - Electrical restitution - Membrane current - Reverse rate dependence - Ventricular repolarization

Several cardioactive agents are known to display direct or reverse rate-dependent effects on action potential duration (APD) depending on the experimental conditions. In spite of the several theories developed so far to explain the frequency-dependent nature of drug-effects on APD, the underlying mechanism has not yet been clarified (Hondeghem and Katzung 1977; Jurkiewicz and Sanguinetti 1993; Yang and Roden 1996; Rocchetti et al. 2001; Virág et al. 2009). Recently, a new theory has been proposed suggesting that the reverse ratedependent mode of drug-action may be a common property of canine, guinea pig, and human cardiac tissues, and this phenomenon is based on the dependence of drug-action on baseline APD (Bányász et al. 2009; Bárándi et al. 2010). The

Correspondence to: Péter P. Nánási, Department of Physiology, University of Debrecen, Nagyerdei krt 98, H-4012 Debrecen, Hungary

E-mail: nanasi@phys.dote.hu aim of the present work was to examine the limitations of this hypothesis by studying the APD lengthening effect of $\mathrm{K}^{+}$channel blockers and the APD shortening effect of $\mathrm{Ca}^{2+}$ channel blockers during the electrical restitution process of rat ventricular action potentials. Rat ventricular muscle was chosen because it has a set of ion currents markedly different from those of other species, and consequently, its APD is shorter by one order of magnitude than that of the "plateauforming" larger mammals (Josephson et al. 1984; Apkon and Nerbonne 1991; Yuan et al. 1996). But, most importantly, because rat ventricular APD shortens with decreasing the pacing frequency - in contrast to many other mammalian species (Carmeliet 1977; Szigligeti et al. 1996; Shigematsu et al. 1997). The restitution of APD was studied as a function of the diastolic interval, a parameter indicating the proximity of action potentials. Using this model we hope to answer the following specific questions: 1) Is the above defined dependence of drug-effects on baseline APD evident only 
in case of reverse rate-dependence, or also under conditions resulting direct rate-dependent changes? In other words, is the frequency-dependent nature of drug-effects of APD a direct consequence of changing the stimulation frequency, or is related to the concomitant shift in baseline APD. 2) Is the phenomenon evident only under steady-state conditions, or it can also be observed following abrupt changes of the cycle length (i.e. under conditions of electrical restitution)? 3) Is a long lasting plateau, characteristic of larger mammals, required, or the phenomenon is also present is species having short APD, such are rats and mice? These questions can be answered by studying various drug-effects in rat ventricular myocardium under conditions of electrical restitution, due to the peculiar properties of this preparation. The observed drug-induced changes in APD were proportional with the pre-drug value of APD, rather than the diastolic interval, indicating that not the proximity of consecutive action potentials, but the initial duration of the action potential may determine the magnitude of drug-induced APD changes in rat ventricular myocardium following abrupt changes in diastolic interval.

Adult Wistar rats of either sex were anesthetized with Na-pentobarbital according to a protocol approved by the Animal Care Committee of the University of Debrecen in compliance with the Guide for the Care and Use of Laboratory Animals (USA NIH publication No. 86-23, revised 1985). After opening the chest, the hearts were quickly removed and placed in Tyrode solution containing (in mmol/l): $\mathrm{NaCl} 153 ; \mathrm{KCl} 5.4 ; \mathrm{CaCl}_{2} 2.7 ; \mathrm{MgCl}_{2}$ 1.05; HEPES 5.4; and glucose 11.0. The $\mathrm{pH}$ of this superfusate was $7.4 \pm$ 0.05 at $37^{\circ} \mathrm{C}$ when gassed with $100 \% \mathrm{O}_{2}$. Isolated pieces of ventricular free wall with trabeculae were excised from adult rat hearts. The preparations were individually mounted in a plexiglass chamber allowing for continuous superfusion at a rate of $10 \mathrm{ml} / \mathrm{min}$. The preparations were stimulated using $1 \mathrm{~ms}$ wide isolated constant current pulses having amplitudes of twice the diastolic threshold. These pulses were provided by a computer-controlled stimulator at a constant rate of $1 \mathrm{~Hz}$, and delivered to the preparation through a pair of platinum electrodes. Conventional $3 \mathrm{~mol} / \mathrm{K} \mathrm{KCl}$ filled glass microelectrodes having resistances between 10 and $20 \mathrm{M} \Omega$ were used to record action potentials. After amplification (INTR-01, Experimetria, Budapest, Hungary) the evoked potentials were digitized using a $100 \mathrm{kHz}$ A/D converter (UAM-1500, Debrecen, Hungary) and analyzed offline. Microelectrode impalements were continuously maintained throughout each experimental protocol.

Restitution of APD was studied as follows. The preparations were continuously paced using a train of 20 basic stimuli delivered at a frequency of $1 \mathrm{~Hz}$. These trains of basic stimuli were interrupted by a single extra stimulus applied at successively longer coupling intervals. In this way, each 20th basic action potential was followed by a single extra action potential occurring at successively longer diastolic intervals (DI). DI was defined as a time elapsed from the action potential duration measured at $90 \%$ level of repolarization $\left(\mathrm{APD}_{90}\right)$ of the last basic action potential of the train to the upstroke of the extra action potential. Restitution curves were generated by plotting the duration of each extra action potential measured at $50 \%$ level of repolarization $\left(\mathrm{APD}_{50}\right)$ against the respective $\mathrm{DI}$. Using the $\mathrm{APD}_{50}$ values prevented uncertainty arising from determination of $\mathrm{APD}_{90}$, where the rate of potential change is less sharp due to the pronounced negative afterpotential.

To study the relationship between baseline APD and druginduced APD changes, rat ventricular action potentials were lengthened using the $\mathrm{I}_{\text {to }}$ blocker 4-aminopyridine (4-AP) and the $\mathrm{I}_{\mathrm{K}}$ blocker tetraethylammonium (TEA) (both at a concentration of $5 \mathrm{mmol} / \mathrm{l}$ ), while shortened by application of $2.5 \mu \mathrm{mol} / \mathrm{l}$ nifedipine and $2 \mathrm{mmol} / \mathrm{l} \mathrm{MnCl}_{2}$, both known as $\mathrm{I}_{\mathrm{Ca}}$ blocking agents (Mitchell et al. 1984). Thus, from the four drugs used, TEA and 4-AP act on different types of $\mathrm{K}^{+}$channels, while nifedipine is known to display strong use-dependent interaction with L-type $\mathrm{Ca}^{2+}$ channels - in contrast to the purely voltage-dependent interaction of $\mathrm{MnCl}_{2}$. The applied drug concentrations were sufficiently high to induce significant changes in $\mathrm{APD}_{50}$ under steadystate conditions, measured at a constant pacing frequency of $1 \mathrm{~Hz}$, as presented in Fig. 1.

The restitution relations, i.e. $\mathrm{APD}_{50}$ values plotted against DI, are presented in Fig. 2. APD monotonically decreased in rat ventricular muscle preparations when DI was increased from $50 \mathrm{~ms}$ to $4 \mathrm{~s}$. APD was increased by TEA and 4-AP - both superfused for $60 \mathrm{~min}$ (Figs. $2 \mathrm{~A}$

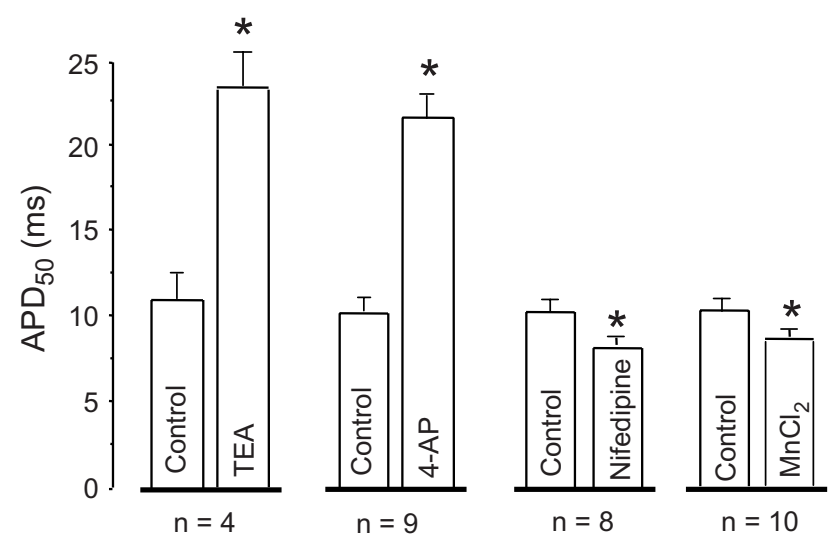

Figure 1. Steady-state effects of $5 \mathrm{mmol} / \mathrm{l} \mathrm{TEA}, 5 \mathrm{mmol} / \mathrm{l} 4$-AP, 2.5 $\mu \mathrm{mol} / \mathrm{l}$ nifedipine, and $2 \mathrm{mmol} / \mathrm{l} \mathrm{MnCl}_{2}$ on action potential duration measured at $50 \%$ level of repolarization $\left(\mathrm{APD}_{50}\right)$ at a constant pacing frequency of $1 \mathrm{~Hz}$. Columns and bars are mean \pm SEM values, number of preparations is indicated as $\mathrm{n}$, and asterisks denote significant changes $(p<0.05)$ determined using Student's $t$-test for paired data. 

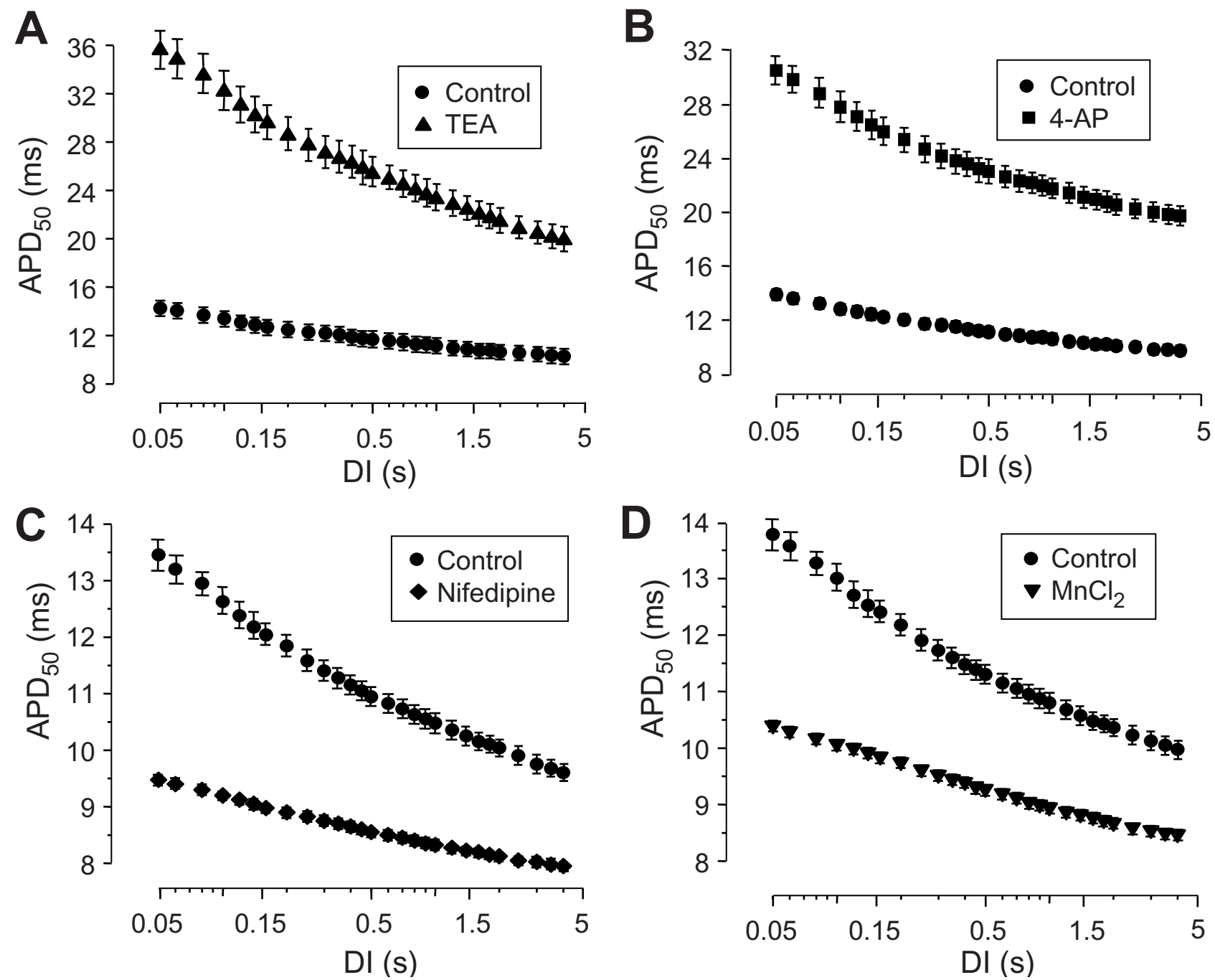

Figure 2. Electrical restitution relations obtained for $\mathrm{APD}_{50}$ in rat ventricular muscles in the presence of $5 \mathrm{mmol} / \mathrm{l}$ TEA $(n=4$; $\mathrm{A})$, $5 \mathrm{mmol} / \mathrm{l} 4-\mathrm{AP}(n=9 ; \mathrm{B}), 2.5 \mu \mathrm{mol} / \mathrm{l}$ nifedipine $(n=8 ; \mathrm{C})$, and $2 \mathrm{mmol} / \mathrm{l} \mathrm{MnCl}_{2}(n=10 ; \mathrm{D})$. Corresponding pre-drug control values are indicated by circles. Basic cycle length was $1 \mathrm{~s}$ in each experiment. Symbols and bars denote mean $\pm \mathrm{SEM}_{\text {values. }} \mathrm{APD}_{50}$, action potential duration measured at $50 \%$ level of repolarization; DI, diastolic interval.

and $\mathrm{B})$. In contrast, APD was shortened by nifedipine and $\mathrm{MnCl}_{2}$ at each DI (Figs. 2C and D). The magnitude of the drug-induced lengthening and shortening of APD was greater at shorter than at longer DI values, as shown in Figs. $3 \mathrm{~A}$ and $\mathrm{B}$, respectively. Plotting these drug-induced APD changes as a function of the respective control (pre-drug) APD indicated that lengthening and shortening of APD was always proportional to the control APD measured before drug treatment - independently of the actual value of DI (Figs. 3C and D). In other words, the APD modifying effects of 4-AP, TEA, nifedipine, and $\mathrm{MnCl}_{2}$ showed positive rate-dependent properties in rat (they were more pronounced at shorter DI values than at longer ones), while their magnitudes were still proportional to the duration of the pre-drug action potential.

The major finding of this study was to show that drug-induced lengthening or shortening of APD is more pronounced when the pre-drug action potential is longer independently of the experimental conditions (i.e., the APD-frequency relationship, duration of the plateau, or the mode of pacing). In canine, guinea pig and human cardiac preparations drug-induced APD changes increased with increasing the cycle length showing reverse rate dependency (Bányász et al. 2009; Bárándi et al. 2010), while in the present study, performed in rat, more pronounced changes were observed with decreasing the DI (direct rate dependency). However, 

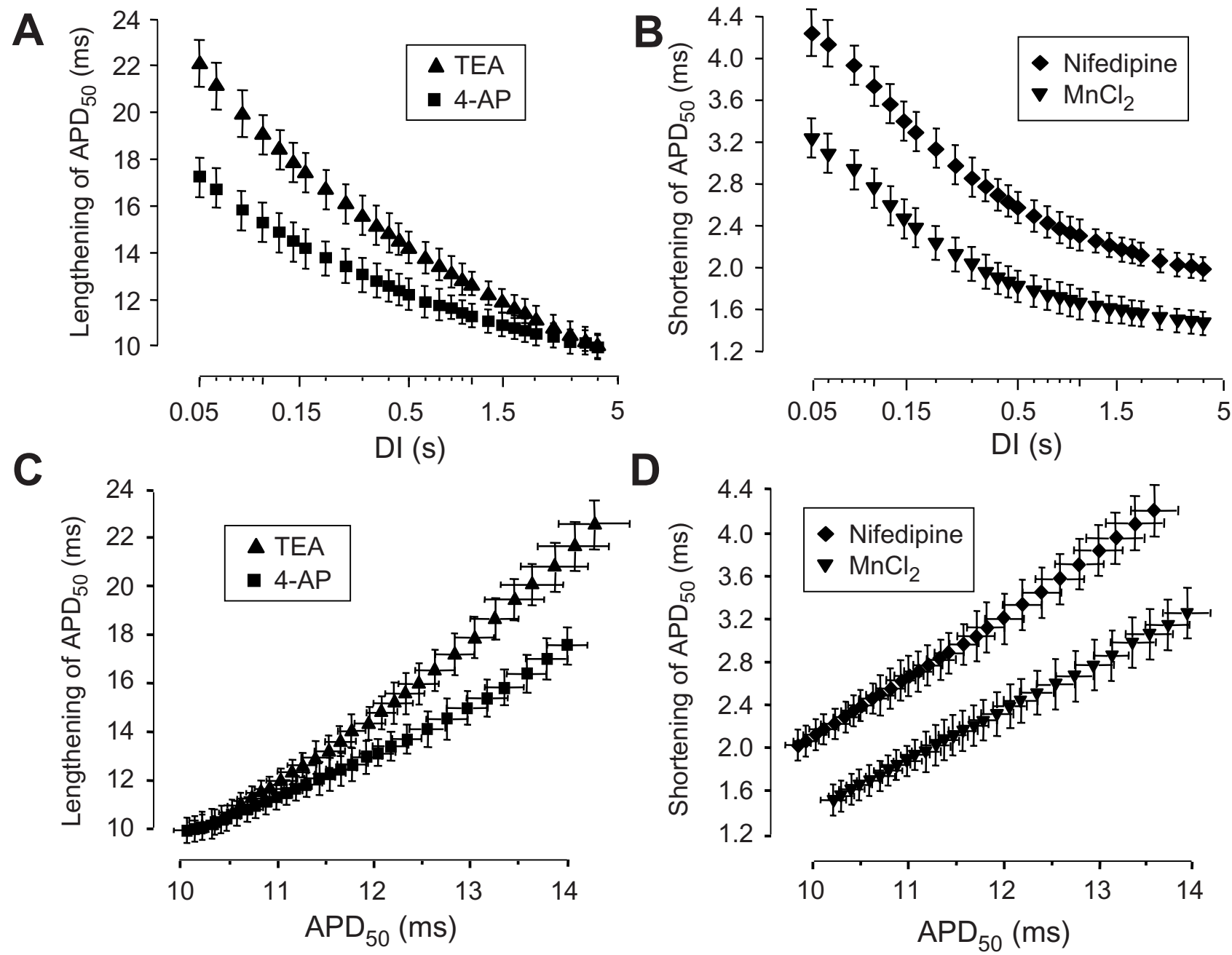

Figure 3. A and B. Drug-induced changes in action potential duration as a function of diastolic interval. C and D. Drug-induced lengthening (C) and shortening (D) of $\mathrm{APD}_{50}$ plotted as a function of the respective control (pre-drug) value of $\mathrm{APD}_{50}$, obtained from the same preparation at the same diastolic interval. Data are obtained from experiments presented in Fig. 2. Symbols and bars denote mean \pm SEM values. $\mathrm{APD}_{50}$, action potential duration measured at $50 \%$ level of repolarization; $\mathrm{DI}$, diastolic interval.

in both cases the larger APD changes were observed when the pre-drug APD was the longer. In rat ventricular muscle, the relation between the drug-induced change in APD and pre-drug APD value was almost linear indicating a strong correlation between the two parameters. However, the existence of an intrinsic dependency of APD modulation on baseline APD does not rule out that other genuinely rate-dependent mechanisms, such as drug-ion channel interactions (Hondeghem and Katzung 1977; Jurkiewicz and Sanguinetti 1993; Yang and Roden 1996) and rate dependency of ion current kinetics (Rocchetti et al. 2001; Virág et al. 2009), may also contribute to determine the magnitude of drug-induced APD changes. These non-uniformities together with differences in the blocking potencies of the drug-concentrations applied may explain the minor differences between the TEA and 4-AP curves or nifedipine and $\mathrm{MnCl}_{2}$ curves observed in Fig. 3 .

In our interpretation both the reverse rate-dependency observed in dog, guinea-pig, and human (Bányász et al. 2009; Bárándi et al. 2010), as well and the positive rate dependency observed in rat (present study) may be a pure consequence of the frequency-dependent behavior of APD itself, namely because APD is longer at higher frequencies in the majority of larger mammals, while it is shorter at higher frequencies in rat. In line with this argumentation, drug effects are expected to be positively rate-dependent in any species responding with shortening of APD to reduced pacing rates. Indeed, levkromakalim was shown to shorten and sotalol to 
lengthen APD in rabbit papillary muscles in a greater extent at shorter than at longer cycle lengths within the range of 0.7-3 s, where APD is decreasing with increasing the cycle length (Szigligeti et al. 1996; Bárándi et al. 2010).

To explain these results it has to be assumed that the net membrane current flowing during the plateau is smaller in the case of a longer than a shorter action potential. In this case the same amount of current blocked by a pharmacological intervention is expected to cause a larger relative shift in the net membrane current, and consequently, a greater change in APD. Therefore, the reverse rate-dependent nature of drug action can be considered as a general and intimate property of all species having positive APD - cycle length relationship (dogs, humans, and guinea pigs), while direct rate-dependency is a characteristic of animals with negative APD - cycle length relationship (rats and mice).

In summary, present results strongly support the theory outlined by Bányász et al. (2009) and Bárándi et al. (2010) suggesting that drug-induced changes of APD are strictly determined by the pre-drug APD value in each mammalian species studied under all experimental conditions.

Acknowledgements. Financial support for the studies was provided by grants from the Hungarian Scientific Research Fund (K-68457, K-73160, CNK-77855). Further support was obtained from the Medical and Health Science Center of University of Debrecen (MEC-14/2008).

\section{References}

Apkon M., Nerbonne J. M. (1991): Characterization of two distinct depolarization-activated $\mathrm{K}+$ currents in isolated adult rat ventricular myocytes. J. Gen. Physiol. 97, 973-1011; doi:10.1085/jgp.97.5.973

Bányász T., Horváth B., Virág L., Bárándi L., Szentandrássy N., Harmati G., Magyar J., Marangoni S., Zaza A., Varró A., Nánási P. P. (2009): Reverse rate dependency is an intrinsic property of canine cardiac preparations. Cardiovasc. Res. 84, 237-244; doi:10.1093/cvr/cvp213

Bárándi L., Virág L., Jost N., Horváth Z., Koncz I., Papp R., Harmati G., Horváth B., Szentandrássy N., Bányász T., Magyar J., Zaza A.,Varró A., Nánási P. P. (2010): Reverse rate-dependent changes are determined by baseline action potential duration in mammalian and human ventricular preparations. Basic Res. Cardiol. 105, 315-323; doi:10.1007/s00395-009-0082-7
Carmeliet E. (1977): Repolarization and frequency in cardiac cells. J. Physiol. (Paris) 73, 903-923

Hondeghem L. M., Katzung B. G. (1977): Time- and voltagedependent interactions of antiarrhythmic drugs with cardiac sodium channels. Biochim. Biophys. Acta 472, 373-398

Josephson I. R., Sanches-Chapula J., Brown A. M. (1984): Early outward current in rat single ventricular cells. Circ. Res. 54, 157-162

Jurkiewicz N. K., Sanguinetti M. C. (1993): Rate-dependent prolongation of cardiac action potentials by a methanesulfonanilide class III antiarrhythmic agent. Specific block of rapidly activating delayed rectifier $\mathrm{K}+$ current by dofetilide. Circ. Res. 72, 75-83

Mitchell M. R., Powell T., Terrar D. A., Twist V. W. (1984): Strontium, nifedipine and 4-aminopyridine modify the time course of the action potential in cells from rat ventricular muscle. Br. J. Pharmacol. 81, 551-556

Rocchetti M., Besana A., Gurrola G.B., Possani L. D., Zaza A. (2001): Rate dependency of delayed rectifier currents during the guinea-pig ventricular action potential. J. Physiol. (London) 534, 721-732; doi:10.1111/j.14697793.2001.00721.x

Shigematsu S., Kiyosue T., Sato T., Arita M. (1997): Rate-dependent prolongation of action potential duration in isolated rat ventricular myocytes. Basic Res. Cardiol. 92, 123-128; doi:10.1007/BF00788629

Szigligeti P., Pankucsi C., Bányász T., Varró A., Nánási P. P. (1996): Action potential duration and force-frequency relationship in isolated rabbit, guinea pig and rat cardiac muscle. J. Comp. Physiol. B 166, 150-155; doi:10.1007/ BF00301179

Virág L., Acsai K., Hála O., Zaza A., Bitay M., Bogáts G., Papp J. G., Varró A. (2009): Self augmentation of the lengthening of repolarization is related to the shape of the cardiac action potential: implication for reverse rate dependency. Br. J. Pharmacol. 156, 1076-1084; doi:10.1111/j.14765381.2009.00116.x

Yang T., Roden D. M. (1996): Extracellular potassium modulation of drug block of IKr. Implications for torsade de pointes and reverse use-dependence. Circulation 93, 407-411

Yuan W., Ginsburg K. S., Bers D. M. (1996): Comparison of sarcolemmal calcium channel current in rabbit and rat ventricular myocytes. J. Physiol. (London) 493, 733-746

Received: February 9, 2010

Final version accepted: April 16, 2010 